CLINICAL STUDY

\title{
Obesity is a risk factor for thyroid cancer in a large, ultrasonographically screened population
}

\author{
Ji Min Han ${ }^{1}$, Tae Yong Kim ${ }^{1}$, Min Ji Jeon ${ }^{1}$, Ji Hye Yim ${ }^{1}$, Won Gu Kim ${ }^{1}$, Dong Eun Song ${ }^{2}$, Suck Joon Hong ${ }^{3}$, \\ Sung Jin Bae ${ }^{4}$, Hong-Kyu Kim ${ }^{4}$, Myung-Hee Shin ${ }^{5}$, Young Kee Shong ${ }^{1}$ and Won Bae Kim ${ }^{1}$ \\ ${ }^{1}$ Department of Internal Medicine, ${ }^{2}$ Department of Pathology, ${ }^{3}$ Department of Surgery and ${ }^{4}$ Health Screening and Promotion Center, Asan Medical Center, \\ University of Ulsan College of Medicine, 388-1 Pungnap-dong, Songpa-gu, Seoul 138-736, Republic of Korea and ${ }^{5}$ Department of Social and Preventive \\ Medicine, School of Medicine, Sungkyunkwan University, Suwon, Republic of Korea
}

(Correspondence should be addressed to W B Kim; Email: kimwb@amc.seoul.kr)

\begin{abstract}
Objective: Obesity is a well-known risk factor for many cancers, including those of the esophagus, colon, kidney, breast, and skin. However, there are few reports on the relationship between obesity and thyroid cancer. We conducted this study to determine whether obesity is a risk factor for thyroid cancer by systematically screening a selected population by ultrasonography.

Design and methods: We obtained data from 15068 subjects that underwent a routine health checkup from 2007 to 2008 at the Health Screening and Promotion Center of Asan Medical Center. Thyroid ultrasonography was included in the checkup, and suspicious nodules were examined by ultrasonography-guided aspiration. Those with a history of thyroid disease or family history of thyroid cancer were excluded from this study.

Results: In total, 15068 subjects, 8491 men and 6577 women, were screened by thyroid ultrasonography. Fine-needle aspiration cytology was performed in 1427 of these patients based on the predefined criteria and thyroid cancer was diagnosed in 267 patients. The prevalence of thyroid cancer in women was associated with a high BMI (per $5 \mathrm{~kg} / \mathrm{m}^{2}$ increase) (odds ratios $(\mathrm{OR})=1.63,95 \%$ CI 1.24-2.10, P<0.001), after adjustment for age, smoking status, and TSH levels. There was no positive correlation between the prevalence of thyroid cancer in men and a high BMI $(\mathrm{OR}=1.16$, $95 \%$ CI $0.85-1.57, P=0.336$ ). There was no association between age, fasting serum insulin, or basal TSH levels and thyroid cancer in either gender.

Conclusions: Obesity was associated with a higher prevalence of thyroid cancer in women when evaluated in a routine health checkup setting. This association between risk factor and disease was unrelated to serum insulin and TSH levels. Additional studies are needed to understand the mechanism(s) behind the association of obesity with thyroid cancer risk.
\end{abstract}

European Journal of Endocrinology 168 879-886

\section{Introduction}

The incidence of thyroid cancer is increasing in many countries $(1,2,3)$. This increase in disease incidence is likely due to an increase in the rate of detection of small papillary carcinomas by widespread use of ultrasonography (1). Nevertheless, Chen et al. (2) reported an increase in thyroid cancer of all sizes between 1988 and 2005 in the United States. This suggested that overdiagnosis is not the only explanation and that other causes such as environmental factor(s) could be involved. A study from Denmark also arrived at a similar conclusion (3). While the use of iodinated salt and an increase in the use of ultrasonography may have contributed to the incidence of thyroid cancer, other risk factors are likely to be involved as such increase started before the introduction of iodinate salt and continues long after implementation of ultrasonography.
The main risk factors for thyroid cancer are exposure to ionizing radiation, a history of benign thyroid disease, and a family history of thyroid cancer $(4,5,6)$. Overweight and obesity, expressed as a high BMI, are possible risk factors for thyroid cancer. According to the National Health and Nutrition Examination Survey (NHANES) of the United States compiled in 2009-2010, the prevalence of obesity was 35.5 and $35.8 \%$ among adult men and women respectively, a significant increase compared with $12 \%$ among men and $17 \%$ among women in 1976-1980 (7, 8). Obesity is associated with the development of many cancers, including those of esophagus, colon, kidney, breast, skin, rectum, and gallbladder (9). However, there are few reports on the relationship between obesity and thyroid cancer and the underlying mechanism is largely unknown $(9,10,11,12)$. 
To our knowledge, a study evaluating obesity as a risk factor for thyroid cancer by systematically screening a selected population with ultrasonography has never been reported. In this study, we investigated the association between obesity and thyroid cancer in a population who underwent routine health checkup in Korea and looked into the possible associations between obesity-related metabolic parameters and thyroid cancer.

\section{Materials and methods}

\section{Study population}

This cross-sectional study included 24935 subjects that underwent a routine health checkup from January 2007 to December 2008 at the Health Screening and Promotion Center of Asan Medical Center in Seoul, Korea. Thyroid ultrasonography was included in each checkup. We selected 17413 subjects (9275 men and 8138 women) that provided details on blood pressure (BP), fasting glucose, cholesterol, triglyceride and insulin levels, waist circumference, bioelectrical impedance analysis, and smoking status as part of the health checkup. Subjects with serum TSH levels $<0.4$ or $>10 \mathrm{mU} / \mathrm{l}$ were excluded. If a subject received two or more health checkups during the study periods, only the first one was included in the analysis. Of 17413 subjects, those with a history of thyroid disease (thyroid dysfunction, $n=799$; thyroid nodule, $n=648$; thyroid cancer, $n=10$; surgery on thyroid, $n=154$; medication for thyroid disease, $n=466$ ) and a family history (first-degree relatives only) of thyroid cancer $(n=1142)$ were also excluded. The final analysis included 15068 subjects. We reviewed medical records and analyzed risk factors for thyroid cancer separately in men and women. The local ethics committee approved the study protocol.

\section{Biochemical measurements}

Venous blood was collected from 12-h fasting subjects in the morning and analyzed for glucose, cholesterol, triglyceride, and insulin at the Asan Medical Center. Glucose, cholesterol, and triglyceride levels were quantified with a TBA-200FR autoanalyzer (Toshiba Medical System Co., Ltd, Tokyo, Japan), whereas insulin was measured by RIA (Insulin IRMA kit; Izotop, Budapest, Hungary). The normal range of insulin was $0.9-8.5 \mathrm{mU} / \mathrm{l}$. Insulin resistance was determined by the homeostasis model assessment method-insulin resistance (HOMA-IR) using fasting glucose and insulin levels as baselines (13). HOMA-IR was calculated by dividing the product of glucose $(\mathrm{mg} / \mathrm{dl})$ and insulin $(\mathrm{mU} / \mathrm{l})$ by 405. Serum TSH was measured by RIA (TSH-CTK-3 kit; DiaSorin S.p.A., Saluggia, Italy). The functional sensitivity of the TSH assay was $0.07 \mathrm{mU} / \mathrm{l}$.

\section{Anthropometric measurements}

During the initial visit, height and weight were obtained with subjects wearing light clothes and no shoes. BMI was calculated as the weight in kilograms divided by the height in meters squared $\left(\mathrm{kg} / \mathrm{m}^{2}\right)$. BMI was categorized as follows: <18.5, 18.5-22.9, $23.0-24.9,25.0-29.9$, and $\geq 30$ for underweight, normal-weight, overweight, obesity, and severe obesity respectively, according to WHO guidelines for Asians (14). BP was measured after at least $30 \mathrm{~min}$ of resting. Waist circumference was measured midway between the costal margin and the iliac crest at the end of a normal expiration. Bioelectrical impedance analysis was performed using an eight-polar tactile-electrode impedance meter, the body composition analyzer (InBody 3.0; Biospace, Seoul, Korea). Resistance of the arms, trunk, and legs to a small alternating electrical current was measured at frequencies of 5,50 , and $250 \mathrm{kHz}$. Total body water and fat-free mass were estimated automatically from the measured resistance using a built-in software. Fat mass was calculated as body weight minus fat-free mass and fat ratio as fat mass divided by body weight.

\section{Identification of thyroid cancer}

Of 15068 subjects, 7472 patients had cystic or solid nodules on thyroid ultrasonography. Patients with abnormal thyroid ultrasonography findings were referred to as endocrinologists, regardless of nodule size or characteristics. Fine-needle aspiration (FNA) cytology was performed on 1427 patients (out of 7472) with thyroid nodule(s) larger than $1 \mathrm{~cm}$ or smaller than $1 \mathrm{~cm}$ when micro-calcification, hypo-echogenicity, a 'taller than wide' shape, irregular margins, or neck lymph node enlargement were noted by thyroid ultrasonography (15). Two hundred and sixty-nine patients were diagnosed as suspicious for malignancy or malignancy on FNA cytology (16). Of 269 subjects, 206 patients underwent thyroid surgery at the Asan Medical Center. The final diagnosis was papillary carcinoma for 203 subjects, follicular carcinoma for one subject, and benign growth for two subjects. We regarded 63 patients who were diagnosed with "papillary thyroid carcinoma' (PTC) by FNA cytology but did not undergo surgery at Asan Medical Center, as having thyroid cancer. Among those 63 patients, 47 underwent surgery in other centers and confirmed as cancers, seven are under observation without surgery, and we do not know the final status in nine patients. Therefore, this study employed 267 thyroid cancer patients.

\section{Statistical analysis}

Categorical variables were presented as numbers and percentages. Continuous variables were expressed as means (s.D.) or medians (interquartile range). 
Comparisons of continuous variables were performed by Student's t-test. Trends across BMI categories were performed by a $\chi^{2}$ test. Quartiles of variables were calculated based on the values from a sex-specific population. R (version 2.12) and R library survivals (R Foundation for Statistical Computing, Vienna, Austria; http://www.R-project.org) were used for statistical analysis. The association between variables and thyroid cancer risk was presented as an odds ratio (OR) with a 95\% CI calculated by binominal logistic regression. $P<0.05$ was considered statistically significant.

\section{Results}

\section{Baseline characteristics of healthy and cancer subjects}

A total of 15068 subjects (8491 men and 6577 women) without any history of thyroid disease were included in this study. A comparison of clinical characteristics between healthy subjects and cancer subjects showed significant differences in all variables between men and women (Table 1). Therefore, data were analyzed separately based on gender. Male subjects with thyroid cancer were significantly younger than subjects without cancer. Female cancer subjects had a higher BMI than non-cancer subjects. The percentage of obese subjects $(\mathrm{BMI} \geq 25)$ was greater in the female cancer group vs the normal group (30 vs $22 \%$ ). In addition, mean waist circumference, mid-BP, and fat ratio were significantly higher in the female cancer group than in the non-cancer group (Table 1).

\section{Subgroup analysis: clinicopathological characteristics of the 204 patients who were diagnosed with thyroid cancer confirmed by surgery in Asan Medical Center}

Of 269 patients with results of suspicious for malignancy or malignancy on cytology, 206 patients underwent thyroid surgery at the Asan Medical Center. The final diagnosis was papillary or follicular carcinoma for 204 patients (98 men and 106 women) and benign growth for two subjects. The clinicopathological characteristics of these 204 cases are presented in Table 2. The median time from health checkup to surgery was 8.4 months, the mean age was 52 years old and $52 \%$ of subjects were women. The median size of tumors was $0.6 \mathrm{~cm}$ $(0.4-1.0)$ and $76 \%$ were $<1 \mathrm{~cm}$ in maximal diameter. The percentage of papillary (including classical PTC and follicular variants), follicular, and mixed carcinomas were $98.5,0.5$, and $1 \%$ respectively; thus, most cancers were papillary thyroid microcarcinomas (PTMCs). The frequency of extrathyroidal extension, presence of central neck lymph node metastasis, and presence of lateral neck lymph node metastasis were 58, 34, and $7 \%$ respectively. According to the TNM staging system (a classification of the International Union Against Cancer and the American Joint Committee on Cancer, revised in 2009), 88 patients were at stage I/II and 116 patients were at stage III/IV (Table 2).

\section{Association between variables and thyroid cancer risk}

We examined the associations between obesity-related metabolic parameters and thyroid cancer (Table 3).

Table 1 Comparison of clinical characteristics between subjects with and without thyroid cancer.

\begin{tabular}{|c|c|c|c|c|c|c|}
\hline & \multicolumn{3}{|c|}{ Male } & \multicolumn{3}{|c|}{ Female } \\
\hline & $\begin{array}{l}\text { Non-cancer } \\
(n=8364)\end{array}$ & $\begin{array}{l}\text { Cancer } \\
(n=127)\end{array}$ & $P$ value & $\begin{array}{c}\text { Non-cancer } \\
(n=6437)\end{array}$ & $\begin{array}{l}\text { Cancer } \\
(n=140)\end{array}$ & $P$ value \\
\hline Age (years) & $52.4 \pm 9.0$ & $50.8 \pm 8.3$ & 0.028 & $51.1 \pm 9.3$ & $50.4 \pm 9.0$ & 0.434 \\
\hline Smoking & $6823(82 \%)$ & $103(81 \%)$ & 0.278 & $591(9 \%)$ & $7(5 \%)$ & 0.327 \\
\hline BMI $\left(\mathrm{kg} / \mathrm{m}^{2}\right)$ & $24.7 \pm 2.8$ & $25.0 \pm 3.0$ & 0.295 & $22.9 \pm 3.0$ & $23.7 \pm 3.2$ & 0.004 \\
\hline$<18.5$ (underweight) & $88(1 \%)$ & $1(1 \%)$ & & $268(4 \%)$ & $4(3 \%)$ & \\
\hline 18.5-22.9 (normal-weight) & 2003 (24\%) & $29(23 \%)$ & & 3256 (51\%) & $54(39 \%)$ & \\
\hline 23.0-24.9 (overweight) & $2468(30 \%)$ & $37(29 \%)$ & & $1505(23 \%)$ & $39(28 \%)$ & \\
\hline $25.0-29.9$ (obesity) & $3533(42 \%)$ & $52(41 \%)$ & & $1271(20 \%)$ & $37(26 \%)$ & \\
\hline$\geq 30.0$ (severe obesity) & $272(3 \%)$ & $8(6 \%)$ & & $137(2 \%)$ & $6(4 \%)$ & \\
\hline Height $(\mathrm{cm})$ & $169.9 \pm 5.7$ & $170.7 \pm 5.6$ & 0.152 & $157.5 \pm 5.4$ & $157.2 \pm 5.5$ & 0.500 \\
\hline Waist circumference $(\mathrm{cm})$ & $86.7 \pm 7.5$ & $87.1 \pm 7.6$ & 0.525 & $77.1 \pm 8.1$ & $79.4 \pm 8.3$ & 0.002 \\
\hline Fat ratio (\%) & $19.6 \pm 4.6$ & $19.6 \pm 4.5$ & 0.849 & $25.9 \pm 5.7$ & $27.3 \pm 6.1$ & 0.008 \\
\hline Mid-BP $(\mathrm{mmHg})$ & $96.7 \pm 8.5$ & $98.0 \pm 7.6$ & 0.064 & $91.1 \pm 10.0$ & $93.7 \pm 9.8$ & 0.002 \\
\hline Glucose (mg/dl) & $101(94-111)$ & $100(95-109)$ & 0.664 & $95.0(89-102)$ & $98.5(90-107)$ & 0.066 \\
\hline Insulin (mIU/l) & $6.5(4.6-9.4)$ & $6.5(4.6-9.2)$ & 0.991 & $5.8(4.1-8.4)$ & $6.5(4.6-9.1)$ & 0.201 \\
\hline HOMA-IR & $1.7(1.1-2.5)$ & $1.6(1.1-2.6)$ & 0.758 & $1.4(0.9-2.1)$ & $1.6(1.1-2.2)$ & 0.098 \\
\hline Cholesterol (mg/dl) & $191.6 \pm 33.9$ & $193.3 \pm 31.3$ & 0.533 & $193.6 \pm 35.2$ & $192.8 \pm 36.8$ & 0.798 \\
\hline Triglycerides (mg/dl) & $130(93-185)$ & $139(102-186)$ & 0.650 & $92(67-129)$ & $90(68-138)$ & 0.159 \\
\hline TSH $(\mathrm{mU} / \mathrm{l})$ & $2.1(1.4-3.1)$ & $2.0(1.4-3.0)$ & 0.831 & $2.5(1.7-3.6)$ & $2.4(1.6-3.4)$ & 0.468 \\
\hline
\end{tabular}

Mid-BP, mid blood pressure $=($ systolic BP + diastolic BP $) / 2 ; \mathrm{HOMA}-\mathrm{IR}$, homeostasis model assessment method-insulin resistance. 
Table 2 Clinicopathological characteristics of patients $(n=204)$ diagnosed with thyroid cancer and confirmed by surgery at the Asan Medical Center.

\begin{tabular}{lc}
\hline Characteristics & Patients \\
\hline Age (years) & \\
Mean \pm s.D. & $51.8 \pm 9.2$ \\
Gender & \\
Men & $98(48 \%)$ \\
Women & $106(52 \%)$ \\
Pathology & \\
Papillary, classical variant & $198(97 \%)$ \\
Papillary, follicular variant & $3(1.5 \%)$ \\
Follicular & $1(0.5 \%)$ \\
Mixed & $2(1 \%)$ \\
Operation & \\
Lobectomy \pm isthmectomy & $72(35 \%)$ \\
Total thyroidectomy & $132(65 \%)$ \\
Maximal tumor diameter (cm) & \\
Median (IQR) & $0.6(0.4-1.0)$ \\
$\leq 1$ cm & $154(76 \%)$ \\
Extrathyroidal extension & \\
Microscopic & $110(54 \%)$ \\
Gross & $8(4 \%)$ \\
Lymph node metastasis & \\
pNO/Nx & $120(59 \%)$ \\
pN1a & $69(34 \%)$ \\
pN1b & $15(7 \%)$ \\
AJCC TNM stage (2009) & \\
I/II & $88(43 \%)$ \\
III/IV & $116(57 \%)$ \\
\hline
\end{tabular}

IQR, interquartile range.

A total of 267 patients were included in risk factor analysis (204 patients with surgically confirmed thyroid cancer and 63 patients with malignant cytology without surgical intervention). Before adjusting for other factors, the prevalence of thyroid cancer $(\mathrm{OR}=$ $1.51,95 \%$ CI $1.17-1.94, P=0.001)$ was associated with a high BMI (per $5 \mathrm{~kg} / \mathrm{m}^{2}$ ) in women; however, a high BMI did not correlate with the prevalence of thyroid cancer in men $(\mathrm{OR}=1.20,95 \%$ CI $0.87-1.61$, $P=0.252$ ). We also examined this relationship using BMI quartiles. The risk for developing thyroid cancer in women was higher in the third (41\%) and fourth (49\%) quartile groups than in the first quartile group. In addition, mid-BP and the serum fasting glucose level were positively correlated with thyroid cancer only in the fourth vs the first quartile among women. Height, waist circumference, fat ratio, serum insulin level, insulin resistance (HOMA-IR), fasting cholesterol, and triglyceride levels were not independent risk factors for thyroid cancer in either gender.

We selected potential risk factors for thyroid cancer: age, smoking status, serum TSH level, and BMI, to analyze their association with thyroid cancer (Fig. 1). After adjusting for age, smoking status, and serum TSH level, BMI (modeled continuously, per $5 \mathrm{~kg} / \mathrm{m}^{2}$ increase) remained a significant predictor of thyroid cancer $(\mathrm{OR}=1.63,95 \%$ CI 1.24-2.10, $\mathrm{P}<0.001)$ in women. In men, OR for thyroid cancer based on a high BMI was 1.16 (95\% CI $0.85-1.57, P=0.336)$, but this result was not statistically significant. The continuous rise in thyroid cancer prevalence was examined based on increasing BMI categories in both genders (Fig. 2). The prevalence of thyroid cancer, unadjusted for other factors, in underweight, normal-weight, overweight, obesity and severe obesity was $1.1,1.4,1.5,1.5$, and $2.9 \%$ in men and $1.5,1.6,2.5,2.8$, and $4.2 \%$ in women respectively.

\section{Discussion}

In this study, we confirmed that obesity was associated with a higher prevalence of thyroid cancer in subjects that underwent thyroid ultrasonography at one healthcare facility. Our data do not represent the general population because we excluded subjects that had a history or family history of thyroid disease(s), which is partly supported by the very high proportion of papillary vs follicular thyroid cancers found in this sample set. However, examinees might have a tendency to choose a checkup program including thyroid ultrasonography more frequently if they have such problem(s), so we excluded those examinees to minimize selection bias. By doing so, we might have excluded a few cases at more advanced stages of thyroid cancer detected clinically, however, as the number of excluded cases due to history of thyroid cancer was low $(n=10)$, this probably did not affect the results significantly.

We observed a positive correlation between BMI and thyroid cancer prevalence in women, but not in men, and this finding is consistent with previous studies. A pooled analysis of 12 case-control studies showed a strong association with a high BMI in women (OR 1.2, $P=0.04$ ) but not in men (10). A large European prospective study showed an overall $13 \%$ increase in thyroid cancer risk based on an increase in BMI in women $(P=0.025)$, but not in men $(11)$. In a similar European prospective study, BMI associated positively with thyroid cancer only in women (17). On the other hand, Kitahara et al. (18) reported a pooled analysis of five prospective studies and concluded that BMI was associated with thyroid cancer risk in both men (HR 1.21, 0.97-1.49) and women (HR 1.16, 1.08-1.24). While the prevalence of thyroid cancer increased markedly in the severe obesity group in men, prevalence increased continuously with increasing BMI in women in our study. This might suggest that obesity has more profound effects on the development of thyroid cancer in women than in men; however, a reason for these gender differences is not completely understood.

This cross-sectional study has several limitations in its examination of potential risk factors for thyroid cancer. The etiologically relevant period from exposure of potential risk factor to the time of development of thyroid cancer is needed to identify a causal relationship definitely. In our study, parameters of obesity and metabolic syndrome were collected at the same time 
Table 3 Associations between obesity-related metabolic parameters and prevalence of thyroid cancer.

\begin{tabular}{|c|c|c|c|c|}
\hline & \multicolumn{2}{|c|}{ Men } & \multicolumn{2}{|l|}{ Women } \\
\hline & Unadjusted OR $(95 \% \mathrm{Cl})$ & $P$ value & Unadjusted OR $(95 \% \mathrm{Cl})$ & $P$ value \\
\hline BMl (per $\left.5 \mathrm{~kg} / \mathrm{m}^{2}\right)$ & $1.20(0.87-1.61)$ & 0.252 & $1.51(1.17-1.94)$ & 0.001 \\
\hline \multicolumn{5}{|l|}{ BMI $\left(\mathrm{kg} / \mathrm{m}^{2}\right)^{\mathrm{a}}$} \\
\hline $\begin{array}{l}\text { Q2 } \\
\text { Q3 }\end{array}$ & $\begin{array}{l}1.02(0.61-1.72) \\
1.07(0.64-1.79)\end{array}$ & 0.931 & $\begin{array}{l}1.19(0.67-2.12) \\
183(1.10-3.14)\end{array}$ & $\begin{array}{l}0.555 \\
0.023\end{array}$ \\
\hline Q4 & $1.23(0.76-2.03)$ & 0.403 & $2.21(1.35-3.73)$ & $\begin{array}{l}0.023 \\
0.002\end{array}$ \\
\hline \multicolumn{5}{|l|}{ Height $(\mathrm{cm})^{\mathrm{b}}$} \\
\hline Q2 & $0.82(0.48-1.37)$ & 0.447 & $0.87(0.53-1.44)$ & 0.589 \\
\hline Q3 & $0.90(0.56-1.46)$ & 0.683 & $0.97(0.61-1.55)$ & 0.887 \\
\hline Q4 & $0.97(0.60-1.55)$ & 0.890 & $0.74(0.45-1.21)$ & 0.227 \\
\hline \multicolumn{5}{|c|}{ Waist circumference $(\mathrm{cm})^{\mathrm{c}}$} \\
\hline Q2 & $0.98(0.58-1.63)$ & 0.931 & $0.67(0.36-1.22)$ & 0.201 \\
\hline Q3 & $1.06(0.63-1.77)$ & 0.824 & $1.41(0.88-2.27)$ & 0.155 \\
\hline Q4 & $1.13(0.70-1.81)$ & 0.623 & $1.49(0.94-2.40)$ & 0.094 \\
\hline \multicolumn{5}{|l|}{ Fat ratio $(\%)^{d}$} \\
\hline Q2 & $1.00(0.60-1.69)$ & 0.988 & $0.69(0.40-1.17)$ & 0.178 \\
\hline Q3 & $1.48(0.93-2.39)$ & 0.106 & $0.95(0.58-1.55)$ & 0.831 \\
\hline Q4 & $0.84(0.49-1.45)$ & 0.538 & $1.49(0.97-2.34)$ & 0.074 \\
\hline \multicolumn{5}{|l|}{$\operatorname{Mid}-\mathrm{BP}(\mathrm{mmHg})^{\mathrm{e}}$} \\
\hline Q2 & $0.89(0.52-1.53)$ & 0.683 & $0.80(0.46-1.38)$ & 0.426 \\
\hline Q3 & $1.02(0.60-1.71)$ & 0.95 & $1.28(0.79-2.08)$ & 0.323 \\
\hline Q4 & $1.44(0.90-2.33)$ & 0.134 & $1.60(1.02-2.57)$ & 0.045 \\
\hline \multicolumn{5}{|l|}{ Glucose $(\mathrm{mg} / \mathrm{dl})^{\mathrm{f}}$} \\
\hline Q2 & $0.82(0.49-1.34)$ & 0.428 & $1.19(0.69-2.07)$ & 0.533 \\
\hline Q3 & $0.83(0.51-1.32)$ & 0.425 & $1.43(0.86-2.43)$ & 0.174 \\
\hline Q4 & $0.76(0.46-1.23)$ & 0.260 & $1.97(1.23-3.23)$ & 0.006 \\
\hline \multicolumn{5}{|l|}{ Insulin $\left(\mathrm{mU} / \mathrm{l}^{\mathrm{g}}\right.$} \\
\hline Q2 & $0.94(0.57-1.54)$ & 0.795 & $0.76(0.45-1.26)$ & 0.288 \\
\hline Q3 & $0.95(0.58-1.56)$ & 0.853 & $1.05(0.66-1.68)$ & 0.836 \\
\hline Q4 & $0.92(0.56-1.52)$ & 0.750 & $1.15(0.73-1.82)$ & 0.556 \\
\hline \multicolumn{5}{|l|}{ HOMA-IR ${ }^{h}$} \\
\hline Q2 & $0.86(0.53-1.39)$ & 0.547 & $0.94(0.55-1.58)$ & 0.802 \\
\hline Q3 & $0.64(0.38-1.07)$ & 0.095 & $1.38(0.86-2.23)$ & 0.187 \\
\hline Q4 & $0.92(0.57-1.47)$ & 0.716 & $1.38(0.86-2.23)$ & 0.189 \\
\hline \multicolumn{5}{|l|}{ Cholesterol $(\mathrm{mg} / \mathrm{dl})^{\mathrm{i}}$} \\
\hline Q2 & $0.88(0.52-1.48)$ & 0.630 & $1.26(0.79-2.04)$ & 0.330 \\
\hline Q3 & $1.07(0.65-1.76)$ & 0.792 & $1.05(0.64-1.71)$ & 0.857 \\
\hline Q4 & $1.17(0.73-1.87)$ & 0.518 & $1.12(0.69-1.82)$ & 0.651 \\
\hline \multicolumn{5}{|l|}{ Triglyceride $(\mathrm{mg} / \mathrm{dl})^{\mathrm{j}}$} \\
\hline Q2 & $0.66(0.39-1.12)$ & 0.128 & $0.87(0.54-1.40)$ & 0.570 \\
\hline Q3 & $1.01(0.63-1.61)$ & 0.979 & $0.69(0.42-1.14)$ & 0.151 \\
\hline Q4 & $0.94(0.58-1.52)$ & 0.793 & $1.12(0.72-1.75)$ & 0.603 \\
\hline
\end{tabular}

$\mathrm{OR}$, odds ratio; Mid-BP, mid blood pressure = (systolic BP+diastolic BP)/2; HOMA-IR, homeostasis model assessment method-insulin resistance. ${ }^{a}$ Quartiles for BMI: 13.9-22.9, 23.0-24.6, 24.7-26.3, and 26.4-60.7 kg/ $\mathrm{m}^{2}$ in men; $13.5-20.8,20.9-22.5,22.6-24.5$, and $24.6-39.8 \mathrm{~kg} / \mathrm{m}^{2}$ in women. ${ }^{\mathrm{b}}$ Quartiles for height: $146.0-165.9,166.0-169.9,170.0-173.9$, and $174.0-193.0 \mathrm{~cm}$ in men; $133.0-153.9,154.0-156.9,157.0-160.9$, and $161.0-180.0 \mathrm{~cm}$ in women.

${ }^{\mathrm{C}}$ Quartiles for waist circumference: $56.0-81.9,82.0-86.9,87.0-90.9$, and $91.0-135.0 \mathrm{~cm}$ in men; 33.0-70.9, 71.0-75.9, 76.0-81.9, and 82.0-118.0 cm in women.

dQuartiles for fat ratio: $3.7-16.5,16.6-19.4,19.5-22.4$, and $22.5-53.8 \%$ in men; $5.9-21.8,21.9-25.6,25.7-29.8$, and $29.9-50.0 \%$ in women

${ }^{\mathrm{e}}$ Quartiles for mid-BP: 66.5-90.4, 90.5-97.4, 97.5-103.4, and 103.5-124.0 mmHg in men; 37.5-83.4, 83.5-90.4, 90.5-98.9, and 99.0-119.5 mmHg in women.

${ }^{f}$ Quartiles for glucose: 52-93, 94-100, 101-110, and 111-379 mg/dl in men; 62-88, 89-94, 95-101, and 102-500 mg/dl in women.

${ }^{9}$ Quartiles for insulin: $1.2-4.5,4.6-6.4,6.5-9.3$, and $9.4-181.0 \mathrm{mU} / \mathrm{l}$ in men; $1.4-4.0,4.1-5.7,5.8-8.3$, and 8.4-75.5 $\mathrm{mU} / \mathrm{l}$ in women.

${ }^{\mathrm{h}}$ Quartiles for HOMA-IR: 0.2-1.0, 1.1-1.6, 1.7-2.4, and 2.5-67.0 in men; 0.2-0.8, 0.9-1.3, 1.4-2.0, and 2.1-24.7 in women.

'Quartiles for cholesterol: 66-167, 168-188, 190-212, and 213-473 mg/dl in men; 71-168, 169-190, 191-215, and 216-409 mg/dl in women.

'Quartiles for triglyceride: 31-92, 93-129, 130-184, and 185-1817 mg/dl in men; 24-66, 67-91, 92-128, and 129-901 mg/dl in women.

as thyroid cancer diagnosis. The association between these parameters and thyroid cancer might have been influenced by so-called 'reverse causation'. Nonetheless, this study has several strengths. First, all 15068 subjects underwent thyroid ultrasonography, regardless of subjective symptoms. For this reason, almost all thyroid cancer cases were detected at very early stages, and this might represent the true prevalence of the disease. In previous epidemiological studies, thyroid cancer diagnosis was based on clinical detection rather than routine ultrasonography. This might have underestimated the true prevalence of thyroid cancer. Secondly, this is the first study evaluating potential risk factors for thyroid cancer such as parameters for 

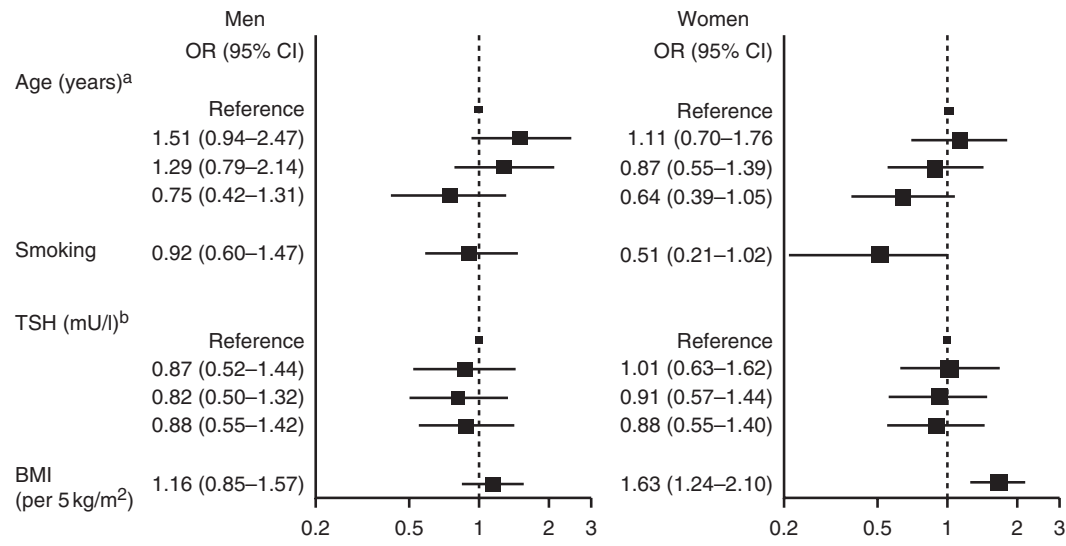

Figure 1 Adjusted OR for the presence of thyroid cancer according to the anthropometric and biochemical variables. ${ }^{\mathrm{a}} \mathrm{Quartiles}$ for age: 19.2-46.2, 46.3-51.9, 52.0-58.2, and 58.3-93.6 years in men; 19.0-44.8, 44.9$50.7,50.8-57.0$, and $57.1-85.7$ years in women. ${ }^{b}$ Quartiles for TSH: 0.4-1.3, 1.4-2.0, 2.1-3.0, and 3.1-10.0 $\mathrm{mU} / \mathrm{l}$ in men; 0.4-1.5, 1.6-2.4, 2.5-3.5, and 3.6-10.0 $\mathrm{mU} / \mathrm{l}$ in women. OR, odds ratio; HOMA-IR, homeostasis model assessment method-insulin resistance. obesity and metabolic syndrome, insulin and TSH levels, and smoking status within a single cohort. As many studies have investigated that the association between obesity and thyroid cancer was epidemiological in nature, serum insulin and TSH levels were not evaluated. Thirdly, thyroid ultrasonography and biochemical data were available from a single time point. Fourthly, we included subjects with a basal TSH value of 0.4-10.0 mU/l to avoid confounding effects of overt thyroid dysfunction on adiposity. Fifthly, we applied the same diagnostic and follow-up strategies for thyroid nodules to avoid selection bias. Many patients were followed at the same healthcare center. The last strength of this study is its large sample size.

The relationship between obesity and carcinogenesis is not yet well understood. Hyperinsulinemia and/or increased levels of insulin-like growth factors have been recognized as potential contributors, especially in colon, breast, endometrial, and pancreatic cancer $(19,20,21)$. However, our study did not support this scenario in thyroid cancer. Serum insulin levels did not show any differences between cancer and healthy subjects in either genders. This means that other mechanism(s) are responsible for the link between obesity and thyroid cancer. White adipose tissue (WAT) represents a source of progenitor cells, which may explain an alternative mechanism for the adverse effects of obesity. Recruitment of adipose stem cells (ASC) into tumors promotes tumor vascularization and growth in mice $(22,23)$. Injection of human WAT CD34+ endothelial progenitor cells contributes to similar effects (24). In addition, obesity increases the circulation of $\mathrm{CD} 34+$ progenitor cells in humans (25). Obesity is a medical condition of WAT excess, which means that a greater reservoir of ASC is available. The role of ASC in supporting tumor neovascularization provides new insights into the mechanisms linking obesity with cancer. Future studies are needed to better understand the role of ASC in obese thyroid cancer patients.

TSH plays a major role in the regulation of thyroid cell growth and several studies have reported an association between TSH and thyroid cancer $(26,27,28)$. However, we found no association between serum TSH levels and thyroid cancer risk. Gerschpacher et al. (29) reported that TSH is not involved in the de novo oncogenesis of PTMCs but instead plays a role in the progression of preexisting cancers. This may explain our results that showed no association of TSH with thyroid cancer because $74 \%$ of thyroid cancer patients were PTMCs (Table 2). Nevertheless, our study did not evaluate the association between serum TSH and thyroid cancer because only subjects with TSH values between 0.4 and $10.0 \mathrm{mU} / \mathrm{l}$ were included.

This study had additional limitations as well. First, the study population was limited to individuals coming in for a voluntary health checkup and was not representative of the general population. Secondly, not all patients with abnormal cytological results underwent surgery at the Asan Medical Center. Sixty-three patients who did not undergo surgery at the Asan Medical Center might have undergone surgery in other hospitals. Some of them might have been diagnosed with benign thyroid disease; thus, thyroid cancer prevalence might have been slightly overestimated. Thirdly, we did not repeatedly measure serum insulin and TSH levels. A single insulin or TSH value might be insufficient for cancer risk analysis. Fourthly, it is unclear how these data can be applied to different

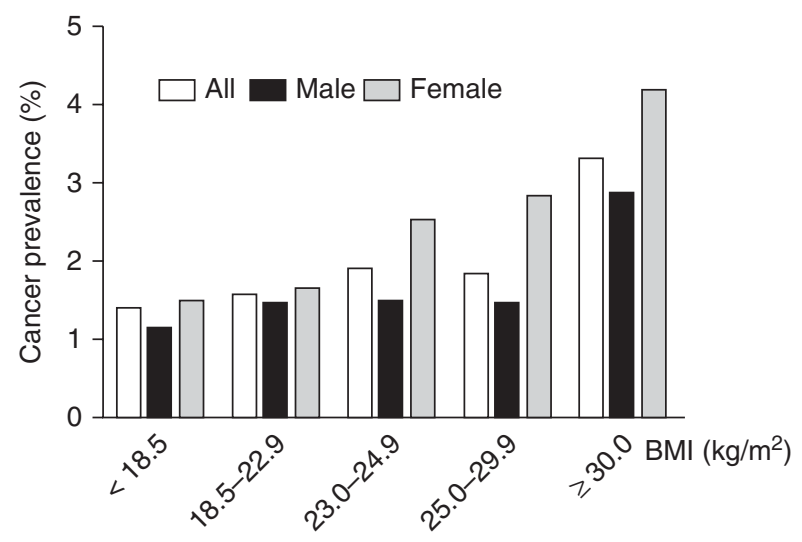

Figure 2 The prevalence of thyroid cancer according to BMI categories. These prevalence estimates are unadjusted for other factors. 
populations, as routine health checkups in most parts of the world do not include thyroid ultrasonography. Many thyroid cancers diagnosed in our study were indolent or clinically insignificant because thyroid cancers were detected at very early stages by thyroid ultrasonography, not by clinical examination. Therefore, the associations between obesity and thyroid cancers in this study may not be applied to clinically significant cancers. Fifthly, obese subjects may be less likely to be detected with thyroid cancer than normal-weight subjects by routine clinical examinations because detection of cancer by palpation may be difficult. So, they had more chance to be diagnosed with thyroid cancer in ultrasonography screening instead of routine clinical examination, which was a potential source of bias. Sixthly, we lacked information on other potential risk factors, such as socioeconomic status, physical activity, alcohol intake, and exogenous hormone use in women. We could not analyze those characteristics and association with thyroid cancer. Despite these limitations, our study clearly showed that obesity was associated with an increase in the prevalence of thyroid cancer. Serum TSH and insulin levels, known as risk factors for cancer, were not related to thyroid cancer.

Obesity is a major public health problem worldwide and its prevalence continues to increase $(7,8)$. The incidence of thyroid cancer has also been increasing in many countries $(1,2)$. Studies on the positive association between obesity and thyroid cancer will have important implications in the future, as obesity is a modifiable risk factor. Future studies on the effects of weight gain or weight loss on altering risk for thyroid cancer are essential.

In conclusion, obesity was associated with a higher prevalence of thyroid cancer in women when evaluated in a routine health checkup setting. This association was not related to either serum insulin or TSH levels. Further large-scale studies are needed to understand the mechanism(s) behind the association of obesity with thyroid cancer risk.

\section{Declaration of interest}

The authors declare that there is no conflict of interest that could be perceived as prejudicing the impartiality of the research reported.

\section{Funding}

This study was supported by the Research Grant Number 2012-0760 of the National Research Foundation of Korea.

\section{References}

1 Davies L \& Welch HG. Increasing incidence of thyroid cancer in the United States, 1973-2002. Journal of the American Medical Association 2006295 2164-2167. (doi:10.1001/jama.295.18. 2164)
2 Chen AY, Jemal A \& Ward EM. Increasing incidence of differentiated thyroid cancer in the United States, 1988-2005. Cancer 2009115 3801-3807. (doi:10.1002/cncr.24416)

3 Blomberg M, Feldt-Rasmussen U, Andersen KK \& Kjaer SK. Thyroid cancer in Denmark 1943-2008, before and after iodine supplementation. International Journal of Cancer 2012131 2360-2366. (doi:10.1002/ijc.27497)

4 Imaizumi M, Usa T, Tominaga T, Neriishi K, Akahoshi M, Nakashima E, Ashizawa K, Hida A, Soda M, Fujiwara S et al. Radiation dose-response relationships for thyroid nodules and autoimmune thyroid diseases in Hiroshima and Nagasaki atomic bomb survivors 55-58 years after radiation exposure. Journal of the American Medical Association 2006295 1011-1022. (doi:10.1001/jama.295.9.1011)

5 Preston-Martin S, Franceschi S, Ron E \& Negri E. Thyroid cancer pooled analysis from 14 case-control studies: what have we learned? Cancer Causes \& Control 200314 787-789. (doi:10.1023/A:1026312203045)

6 Iribarren C, Haselkorn T, Tekawa IS \& Friedman GD. Cohort study of thyroid cancer in a San Francisco Bay area population. International Journal of Cancer 200193 745-750. (doi:10.1002/ ijc.1377)

7 Flegal KM, Carroll MD, Kit BK \& Ogden CL. Prevalence of obesity and trends in the distribution of body mass index among US adults, 1999-2010. Journal of the American Medical Association 2012307 491-497. (doi:10.1001/jama.2012.39)

8 Ljungvall A \& Zimmerman FJ. Bigger bodies: long-term trends and disparities in obesity and body-mass index among U.S. adults, 1960-2008. Social Science \& Medicine 201275 109-119. (doi:10.1016/j.socscimed.2012.03.003)

9 Renehan AG, Tyson M, Egger M, Heller RF \& Zwahlen M. Body-mass index and incidence of cancer: a systematic review and meta-analysis of prospective observational studies. Lancet 2008 371 569-578. (doi:10.1016/S0140-6736(08)60269-X)

10 Dal Maso L, La Vecchia C, Franceschi S, Preston-Martin S, Ron E, Levi F, Mack W, Mark SD, McTiernan A, Kolonel L et al. A pooled analysis of thyroid cancer studies. V. Anthropometric factors. Cancer Causes \& Control 200011 137-144. (doi:10.1023/ A:1008938520101)

11 Rinaldi S, Lise M, Clavel-Chapelon F, Boutron-Ruault M-C, Guillas G, Overvad K, Tjønneland A, Halkjær J, Lukanova A, Kaaks $\mathrm{R}$ et al. Body size and risk of differentiated thyroid carcinomas: findings from the EPIC study. International Journal of Cancer 2012131 E1004-E1014. (doi:10.1002/ijc.27601)

12 Engeland A, Tretli S, Akslen LA \& Bjorge T. Body size and thyroid cancer in two million Norwegian men and women. British Journal of Cancer 200695 366-370. (doi:10.1038/sj.bjc. 6603249)

13 Matthews D, Hosker J, Rudenski A, Naylor B, Treacher D \& Turner R. Homeostasis model assessment: insulin resistance and $\beta$-cell function from fasting plasma glucose and insulin concentrations in man. Diabetologia 198528 412-419. (doi:10.1007/ BF00280883)

14 Consultation WE. Appropriate body-mass index for Asian populations and its implications for policy and intervention strategies. Lancet 2004363 157-163. (doi:10.1016/S0140-6736(03) 15268-3)

15 Cooper DS, Doherty GM, Haugen BR, Kloos RT, Lee SL, Mandel SJ, Mazzaferri EL, McIver B, Sherman SI \& Tuttle RM. Management guidelines for patients with thyroid nodules and differentiated thyroid cancer. Thyroid 200616 109-142. (doi:10.1089/thy. 2006.16.109)

16 Practice TPSoCTFoSo. Guidelines of the Papanicolaou Society of Cytopathology for fine-needle aspiration procedure and reporting. The Papanicolaou Society of Cytopathology Task Force on Standards of Practice. Diagnostic Cytopathology $1997 \quad 17$ 239-247. (doi:10.1002/(SICI)1097-0339(199710)17:4<239::AID-DC1> 3.0.CO;2-7)

17 Almquist M, Johansen D, Bjorge T, Ulmer H, Lindkvist B, Stocks T, Hallmans G, Engeland A, Rapp K, Jonsson H et al. Metabolic 
factors and risk of thyroid cancer in the metabolic syndrome and cancer project (Me-Can). Cancer Causes $\mathcal{E}$ Control 201122 743-751. (doi:10.1007/s10552-011-9747-2)

18 Kitahara CM, Platz EA, Freeman LE, Hsing AW, Linet MS, Park Y, Schairer C, Schatzkin A, Shikany JM \& Berrington de Gonzalez A. Obesity and thyroid cancer risk among U.S. men and women: a pooled analysis of five prospective studies. Cancer Epidemiology, Biomarkers \& Prevention 201120 464-472. (doi:10.1158/10559965.EPI-10-1220)

19 Renehan AG, Roberts DL \& Dive C. Obesity and cancer: pathophysiological and biological mechanisms. Archives of Physiology and Biochemistry 2008114 71-83. (doi:10.1080/ 13813450801954303)

20 Wolin KY, Carson K \& Colditz GA. Obesity and cancer. Oncologist 201015 556-565. (doi:10.1634/theoncologist.2009-0285)

21 Pisani P. Hyper-insulinaemia and cancer, meta-analyses of epidemiological studies. Archives of Physiology and Biochemistry 2008114 63-70. (doi:10.1080/13813450801954451)

22 Zhang Y, Daquinag A, Traktuev DO, Amaya-Manzanares F, Simmons PJ, March KL, Pasqualini R, Arap W \& Kolonin MG. White adipose tissue cells are recruited by experimental tumors and promote cancer progression in mouse models. Cancer Research 200969 5259-5266. (doi:10.1158/0008-5472.CAN-08-3444)

23 Zhang Y, Bellows CF \& Kolonin MG. Adipose tissue-derived progenitor cells and cancer. World Journal of Stem Cells 20102 103-113. (doi:10.4252/wjsc.v2.i5.103)

24 Martin-Padura I, Gregato G, Marighetti P, Mancuso P, Calleri A, Corsini C, Pruneri G, Manzotti M, Lohsiriwat V, Rietjens M et al. The white adipose tissue used in lipotransfer procedures is a rich reservoir of $\mathrm{CD} 34+$ progenitors able to promote cancer progression. Cancer Research 201272 325-334. (doi:10.1158/ 0008-5472.CAN-11-1739)

25 Bellows CF, Zhang Y, Simmons PJ, Khalsa AS \& Kolonin MG. Influence of BMI on level of circulating progenitor cells. Obesity 201119 1722-1726. (doi:10.1038/oby.2010.347)

26 Boelaert K, Horacek J, Holder RL, Watkinson JC, Sheppard MC \& Franklyn JA. Serum thyrotropin concentration as a novel predictor of malignancy in thyroid nodules investigated by fine-needle aspiration. Journal of Clinical Endocrinology and Metabolism 2006 91 4295-4301. (doi:10.1210/jc.2006-0527)

27 Fiore E \& Vitti P. Serum TSH and risk of papillary thyroid cancer in nodular thyroid disease. Journal of Clinical Endocrinology and Metabolism 201297 1134-1145. (doi:10.1210/jc.2011-2735)

28 McLeod DS, Watters KF, Carpenter AD, Ladenson PW, Cooper DS \& Ding EL. Thyrotropin and thyroid cancer diagnosis: a systematic review and dose-response meta-analysis. Journal of Clinical Endocrinology and Metabolism 201297 2682-2692. (doi:10. 1210/jc.2012-1083)

29 Gerschpacher M, Gobl C, Anderwald C, Gessl A \& Krebs M. Thyrotropin serum concentrations in patients with papillary thyroid microcancers. Thyroid 201020 389-392. (doi:10.1089/ thy.2009.0139)

Received 16 November 2012

Revised version received 4 March 2013

Accepted 19 March 2013 